\title{
INVERSOR MULTINIVEL ACOPLADO SIN TRANSFORMADOR A LA LÍNEA ELÉCTRICA PARA LA INCORPORACIÓN DE FUENTES FOTOVOLTAICAS DISTRIBUIDAS
}

\section{GRID CONNECTED INVERTER WITHOUT TRANSFORMER FOR THE INCLUSION OF DISTRIBUTED PHOTOVOLTAIC SOURCES}

\author{
Julio César Viola ${ }^{1, *}$, José Restrepo-Zambrano², José Manuel Aller-Castro ${ }^{2}$ y Flavio \\ Quizhpi-Palomeque ${ }^{1}$
}

\section{Resumen}

El presente trabajo trata sobre el estudio, diseño e implementación de un inversor multinivel capaz de operar con los esquemas de distribución eléctricos más comunes en el Ecuador y que pueda ser acoplado a la línea sin necesidad de transformador. Su control incluye la posibilidad de revertir los flujos de potencia en caso de ser necesaria la carga de bancos de batería. El trabajo presenta los pasos seguidos en el diseño así como las simulaciones de funcionamiento y resultados experimentales preliminares.

Palabras clave: Inversor multinivel, control de flujo de potencia, paneles fotovoltaicos, generación distribuida.

\begin{abstract}
This paper addresses the study, design and implementation of a multilevel inverter aimed to operate with the Ecuador's most commonly used electrical distribution schemes, being able to be connected to the grid without a transformer. The implemented control allows to revert the power flow if it is required (e.g. charge a battery bank). The paper shows the different steps for the design, as well as simulations and preliminary experimental results.
\end{abstract}

Keywords: Multilevel inverter, power flow control, photovoltaic arrays, distributed generation.

\footnotetext{
${ }^{1, *}$ Grupo de Investigación en Energías. Universidad Politécnica Salesiana, Cuenca - Ecuador.

Autor para correspondencia jcviola@iee.org

${ }^{2}$ Universidad Simón Bolívar, Caracas - Venezuela.

Recibido: 15-04-2016, aprobado tras revisión: 31-05-2016.

Forma sugerida de citación: Viola, J.; Restrepo, J.; Aller, J. M.; Quizhpi, F. (2016). «Inversor multinivel acoplado sin transformador a la línea eléctrica para la incorporación de fuentes fotovoltaicas distribuidas». INGENIUS. N. ${ }^{\circ} 15$, (Enero-Junio). pp. 58-65. ISSN: 1390-650X.
} 


\section{Introducción}

El crecimiento vertiginoso de las instalaciones fotovoltaicas domiciliarias durante los últimos años ha sobrepasado incluso a los pronósticos más optimistas. La magnitud de esta expansión se debe fundamentalmente a las políticas de subsidio y reducción de impuestos adoptadas por muchos países donde destacan fundamentalmente: Alemania, China, Italia, España y Estados Unidos (principalmente en el Estado de California). Este fenómeno genera un círculo virtuoso que ha logrado hacer bajar el precio de cada vatio generado por medio de un panel fotovoltaico desde $\$ 77$ en 1977 a menos de $\$ 0.36$ en 2015 [1]. La capacidad mundial total instalada alcanza los $177 \mathrm{GW}$ y se estima que en 2015 se incorporen más de $40 \mathrm{GW}$ adicionales [2] de los cuales se estima que un $20 \%$ será de instalaciones residenciales [1].

La incorporación de este gran número de generadores distribuidos trae consigo problemas de estabilidad dada la impredictibilidad asociada a la generación solar. Para tratar con este problema IEEE redactó en el año 2003 el estándar IEEE1547 titulado «Standard para interconectar recursos distribuidos con los sistemas eléctricos de potencia» [3], [4]. Este estándar sigue siendo actualizado hasta el día de hoy y dentro de sus distintas secciones incluye:

- guías para el diseño y conexión al sistema eléctrico de los inversores y paneles,

- guías para monitoreo, intercambio de información y control de los equipos involucrados, y

- guías para el diseño, operación e integración de sistemas aislados de generación distribuida.

En el Ecuador, la matriz energética está dominada por la generación hidráulica siendo aún bajo el impacto de las instalaciones solares fotovoltaicas, especialmente en lo que se refiere a instalaciones residenciales. La geografía del país y el recurso solar disponible hacen, sin embargo, muy interesante el estudio del impacto que tendría la masificación de instalaciones residenciales basadas en paneles solares. Si se consideran por ejemplo nuevas tendencias de la sociedad como el uso de cocinas de inducción, las cuales imponen un pico de demanda al sistema eléctrico en horas de la mañana, una instalación fotovoltaica domiciliaria de relativo bajo costo podría ayudar a aplanar ese pico. Igualmente el potencial uso masivo de automóviles eléctricos tendría un excelente complemento en el aprovechamiento del recurso solar que cada usuario residencial podría hacer con paneles instalados en el techo de su domicilio.

En el Grupo de Investigación en Energías de la Universidad Politécnica Salesiana se vienen desarrollando en los últimos años numerosos proyectos de investigación en el área de las energías renovables algunos de los cuales han incluido el desarrollo de equipos electrónicos únicos en el país [5], [6]. En esa misma línea de investigación se consideró el realizar un diseño propio para un inversor domiciliario adaptado a la realidad del país en cuanto a costos y normativa eléctrica. En el presente trabajo se describe el estudio, diseño y prueba de un inversor multinivel capaz de ser acoplado a la línea eléctrica sin necesidad de transformador y que permite incorporar de manera segura y estable la generación solar fotovoltaica disponible en instalaciones residenciales. El modo de operación del equipo puede ser fácilmente llevado de monofásico $120 \mathrm{~V}$ a bifásico en topología contrafase el cual constituye el modo más común de suministrar $220 \mathrm{~V}$ domiciliarios en el Ecuador.

La estructura general del artículo es la siguiente: en la sección 2 se describen las instalaciones domiciliarias basadas en paneles fotovoltaicos y sus modos típicos de funcionamiento. En la sección 3 se describen la topología multinivel utilizada, los filtros de acoplamiento a la línea, la capacidad de operación monofásica y bifásica y el diseño de disipadores y gabinete metálico. Finalmente, las secciones 4 y 5 muestran la simulación integral del sistema y algunos resultados experimentales, respectivamente.

\section{Instalaciones residenciales fotovoltaicas}

La estructura típica para una instalación de generación basada en paneles fotovoltaicos se muestra en la Figura 1, donde se pueden observar los paneles fotovoltaicos típicamente instalados sobre el techo de la construcción, el inversor y, opcionalmente, un banco de baterías. El requerimiento o no de poseer banco de baterías depende de las necesidades particulares del usuario, pero también de las legislaciones vigentes en cada país. En algunos casos como los de Japón o Alemania los usuarios están moviéndose al modo de autoconsumo el cual requiere la instalación de baterías las cuales aportan, además, estabilidad al sistema eléctrico [7].

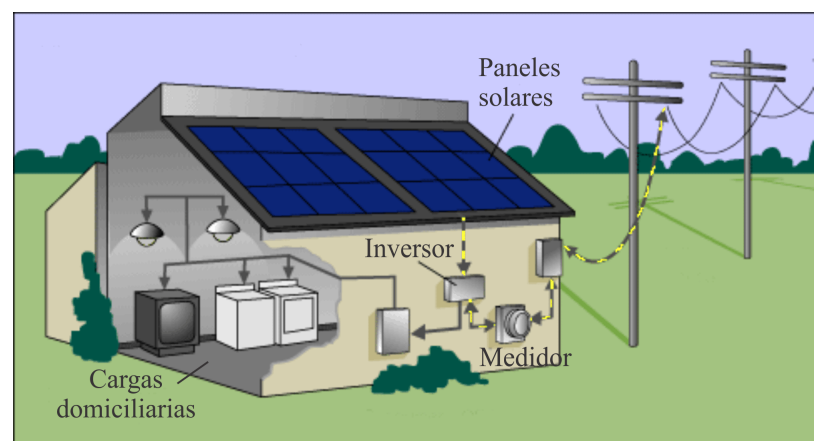

Figura 1. Instalación residencial fotovoltaica típica. 
La conexión de las cargas del usuario se realiza en el punto de acoplamiento común (PCC por sus siglas en inglés) entre el inversor y la red justo «aguas abajo» del medidor de consumo de energía. De esta manera, cuando hay potencia disponible en los paneles fotovoltaicos, el inversor se encargará de extraer toda la potencia disponible en los paneles ante cualquier condición de radiación solar. Para esto el control del inversor se programa con un algoritmo de rastreo del punto de máxima potencia (MPPT por sus siglas en inglés). Esta forma de conexión genera tres escenarios posibles según las potencias de generación disponibles y la demanda establecida por las cargas conectadas:

- La potencia disponible a la salida de los paneles fotovoltaicos $\left(\mathrm{P}_{\mathrm{DC}}\right)$ y en consecuencia a la salida del inversor $\left(\mathrm{P}_{\text {inv }}\right)$ es menor a la demandada por las cargas domiciliarias conectadas. En este caso el inversor ayuda a aliviar el consumo de energía proveniente de la red eléctrica pública que el usuario tendría.

- La potencia $\mathrm{P}_{\mathrm{DC}}$ disponible supera a la requerida por las cargas conectadas en cuyo caso el inversor puede inyectar el excedente hacia la línea pública. Debe observarse que este escenario podría no ser permitido por la legislación en materia eléctrica vigente en algunos países o en algunos casos no estar siquiera normado.

- Un último escenario es el de un corte absoluto del suministro eléctrico en cuyo caso el inversor puede operar en modo isla, esto es desconectándose de la línea eléctrica y alimentando por su cuenta a las cargas domiciliarias.

La Figura 2 resume los tres escenarios descritos, los cuales se toman como guía para el diseño del inversor propuesto en este trabajo ya que se desea que el equipo pueda operar en cada uno ellos. El caso del Escenario 2 es uno de los más interesantes porque permite al usuario «vender» la energía sobrante al sistema eléctrico público en horas de altas radiaciones y «comprar» posteriormente en horas de la noche. Este modo de operación permite que el costo del sistema fotovoltaico se amortice en menos tiempo, ya que no se hace necesario el uso de bancos de baterías para aprovechar los excedentes de generación que existen en ciertos momentos del día.

En el caso del Escenario 3 la norma IEEE1547 requiere que el inversor se desconecte galvánicamente de la línea eléctrica pública para poder seguir alimentando las cargas residenciales. Esto requiere de un algoritmo de detección de modo isla («anti-islanding» en inglés) de manera de asegurar que ante una caída del servicio eléctrico público las líneas no permanezcan energizadas por el inversor lo cual constituye un riesgo para los operarios que potencialmente estén manipulándolas [8].
Escenario 1

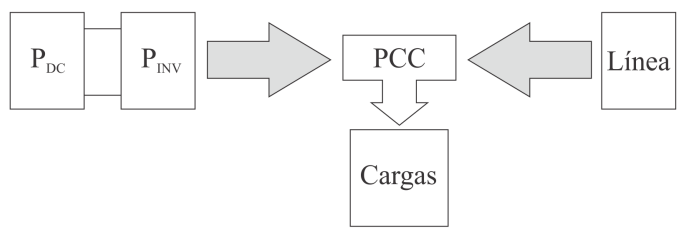

Escenario 2

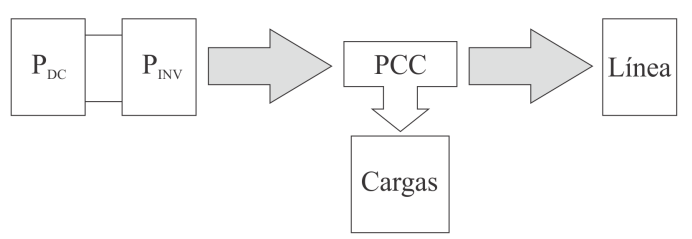

Escenario 3
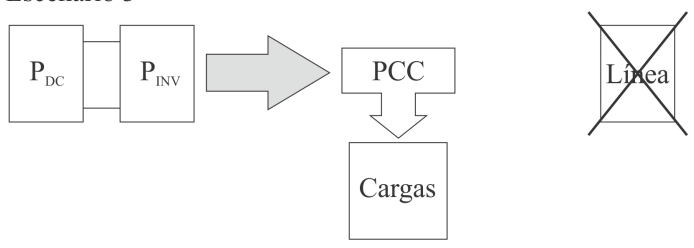

Figura 2. Escenarios resultantes según la potencia generada en los paneles y las cargas conectadas.

\section{Estructura del inversor y su acoplamiento a la red}

Estos dos apartados suelen aparecer juntos en muchos trabajos. No debemos confundir esta discusión o análisis con la obtención de conclusiones, algo que depende tanto de los resultados y de su análisis como del marco teórico y de los objetivos.

\subsection{Topología del inversor multinivel}

El inversor seleccionado es uno de tres niveles de tensión con acoplamiento de punto de neutro (NPC por sus siglas en inglés) cuyo esquema circuital se muestra en la Figura 3. Esta topología fue propuesta en 1981 [9] y es hoy en día la más ampliamente utilizada entre las topologías multinivel. Si bien solo agrega un nivel más de tensión respecto a la clásica estructura de dos niveles posee características que lo hacen muy adecuado para aplicaciones solares tales como el hecho de poder aterrar fácilmente el punto de neutro $(\mathrm{N})$ lo cual es un requerimiento de seguridad dado por la norma IEEE1547. En este diseño se utilizarán transistores bipolares de compuerta aislada (IGBT por sus siglas en inglés) en configuración NPC0. El modelo elegido por su buena relación costo beneficio es el Vincotech 10-FZ07NMA100SM-M265F58 [10], el cual tiene una tensión de bloqueo de $650 \mathrm{~V}$ y maneja corrientes de 100 A. En la sección 3.4 se describe la forma en que estos módulos son fijados a los respectivos disipadores los cuales le permitirán operar a su máxima capacidad de disipación de potencia. 


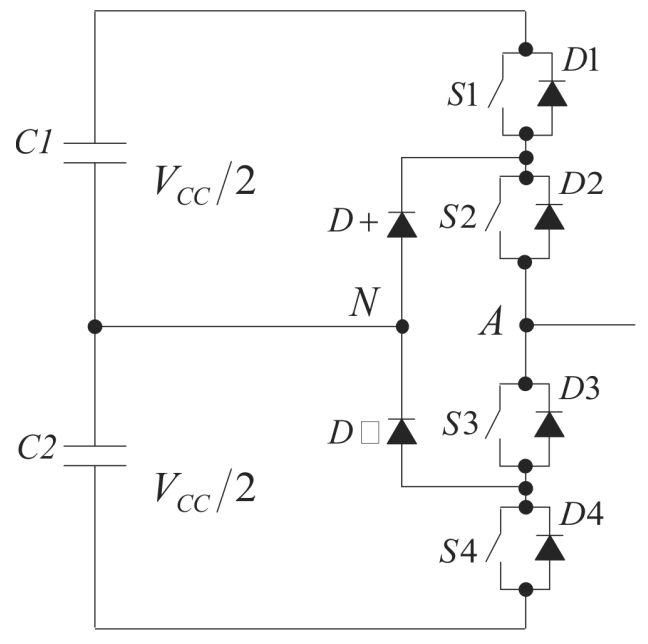

Figura 3. Estructura de inversor 3 niveles con acoplamiento de punto neutro (NPC).

\subsection{Filtros LCL}

El acoplamiento del inversor a la línea eléctrica debe realizarse mediante un elemento que realice un efecto de filtrado o separación entre la tensión sinusoidal presente en la línea y la tensión conmutada entregada por el inversor. Este filtro se encargará de eliminar la mayor parte de los armónicos de corriente de alta frecuencia correspondientes a la frecuencia de conmutación del convertidor dejando pasar los componentes a frecuencia de línea. Los dos esquemas más utilizados son el filtro inductivo que está compuesto de un simple inductor colocado entre el inversor y la tensión de línea y la red LCL la cual se compone de dos inductores y un condensador. Ambos esquemas se muestran en la Figura 4, donde $v_{I N V}$ representa el nodo de tensión del inversor y $v_{L}$ es el nodo de tensión de red.

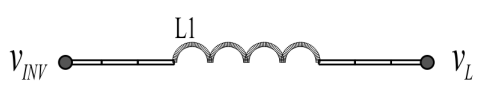

(a)

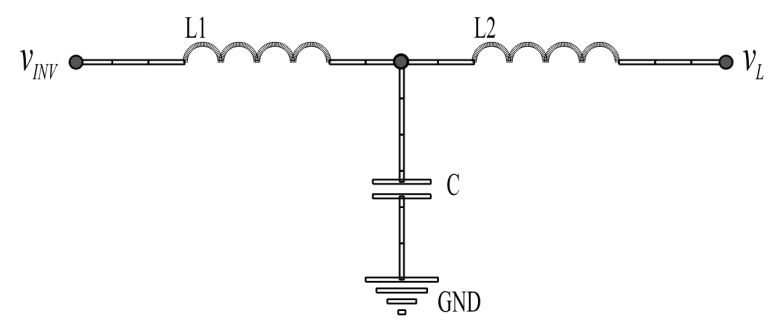

(b)

Figura 4. (a) Filtro inductivo, (b) filtro LCL.

Para el caso del filtro inductivo simple se requiere de valores de inductancia del orden de las decenas de
$\mathrm{mH}$ para poder obtener un filtrado aceptable. Esto implica bobinados relativamente voluminosos, pesados y más caros que los requeridos por el esquema LCL. En este esquema se pueden obtener excelentes resultados con valores para L1 en el orden de las centenas de $\mu \mathrm{H}$ y para L2 en el orden de las decenas de $\mu \mathrm{H}$, en tanto que para el condensador $\mathrm{C}$ se requieren valores del orden de las decenas de $\mu \mathrm{F}$. El volumen, peso y costo de la estructura LCL resulta menor y los efectos de filtrado son mejores dado que la respuesta en frecuencia presenta una pendiente de caída de $-60 \mathrm{~dB} / \mathrm{dec}$ tal como se observa en la Figura 5. Para el caso del filtro puramente inductivo se tienen solamente $-20 \mathrm{~dB} / \mathrm{dec}$. La estructura LCL tiene como desventaja la presencia de un pico de resonancia en $\omega_{0}=\sqrt{(\mathrm{L} 1+\mathrm{L} 2) /(\mathrm{L} 1 \mathrm{~L} 2 C)}$, pero con un adecuado diseño de los componentes puede evitarse que este pico afecte a ninguno de los armónicos involucrados [11]. En el caso del presente diseño la frecuencia de conmutación es de $16 \mathrm{kHz}$ por lo que se diseña para una frecuencia de resonancia $\omega_{0}$ intermedia entre este valor de frecuencia y los armónicos típicos de línea. Un valor de $\omega_{0}=2 \pi * 5.10^{3} \mathrm{rad} / \mathrm{s}$ puede considerarse como una buena relación de compromiso y en ese caso se pueden calcular valores de $\mathrm{L} 1=\mathrm{L} 2=400 \mu H$ y $\mathrm{C}=5 \mu \mathrm{F}$.

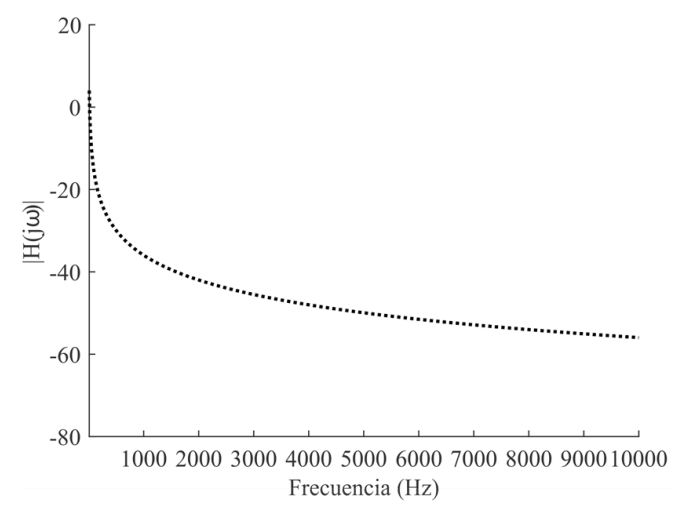

(a)

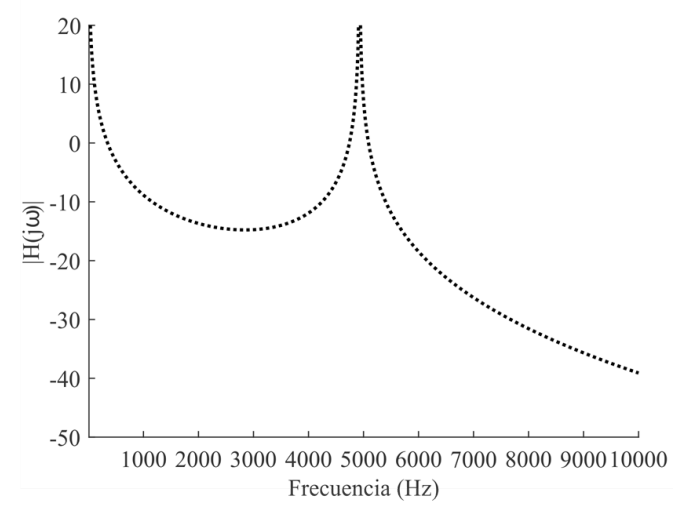

(b)

Figura 5. Respuestas en frecuencia para (a) el filtro puramente inductivo y (b) para el filtro en configuración LCL. 


\subsection{Operación monofásica y bifásica}

El diseño del inversor tiene en cuenta el hecho de que en el Ecuador los dos esquemas de distribución eléctrica domiciliaria más comunes son el monofásico con $120 \mathrm{~V}$ de tensión eficaz nominal entre fase y neutro y el bifásico en configuración contrafase con $220 \mathrm{~V}$ de tensión nominal entre fases. Este último esquema está expandiéndose fuertemente debido al creciente uso de cocinas de inducción las cuales debido a su alto consumo de potencia funcionan en su gran mayoría con $220 \mathrm{~V}$. Se propone entonces una estructura para el inversor que permite fácilmente pasarlo de un formato a otro de funcionamiento con cambios circuitales mínimos. La Figura 6 muestra la estructura diseñada donde mediante un cambio en el control del inversor, el cual es realizado por un microprocesador, se puede hacer operar cada una de las ramas en sincronía o en contrafase. De esta manera, cada rama entrega la potencia nominal calculada para cada IGBT.

\subsection{Diseño de disipadores y gabinete metálico}

Toda la electrónica se monta dentro de un gabinete metálico que le permita ser fácilmente fijado por el instalador pudiendo ser ubicado incluso a la intemperie. Este último requerimiento hace que el material escogido para el gabinete sea aluminio ya que además de la fortaleza mecánica, posee alta inmunidad a la corrosión y también es muy efectivo actuando como jaula de Faraday para las emisiones electromagnéticas típicas de los inversores de potencia. La mayor parte del espacio se dedica a la etapa de potencia la cual incluye a los módulos IGBT, los disipadores y los ventiladores. En la Figura 7 se muestra una sección del gabinete escogido donde se aprecia el montaje de los disipadores de manera longitudinal y los módulos IGBT adosados a cada uno de ellos. Dos requerimientos que son típicamente contradictorios aparecen en este tipo de diseño y es que al mismo tiempo que el gabinete debe permitir la instalación al exterior, se deben diseñar entradas y salidas de aire que permitan hacer circular un flujo de aire apropiado para la refrigeración de todo el sistema. Se decidió colocar entonces las tomas de aire para los ventiladores en la parte inferior del equipo dejando la salida por arriba. La salida se diseña con un sistema de ranuras de manera de que se permite la salida de aire, pero no el ingreso de agua. Adicionalmente se incorporan rejillas que hacen de filtro tanto a la entrada como a la salida de aire para evitar el ingreso de insectos y minimizar el ingreso de polvo a los disipadores. El canal central que queda en medio de los dos disipadores se dedica a toda la electrónica de sensado y control, así como a las fuentes de alimentación.

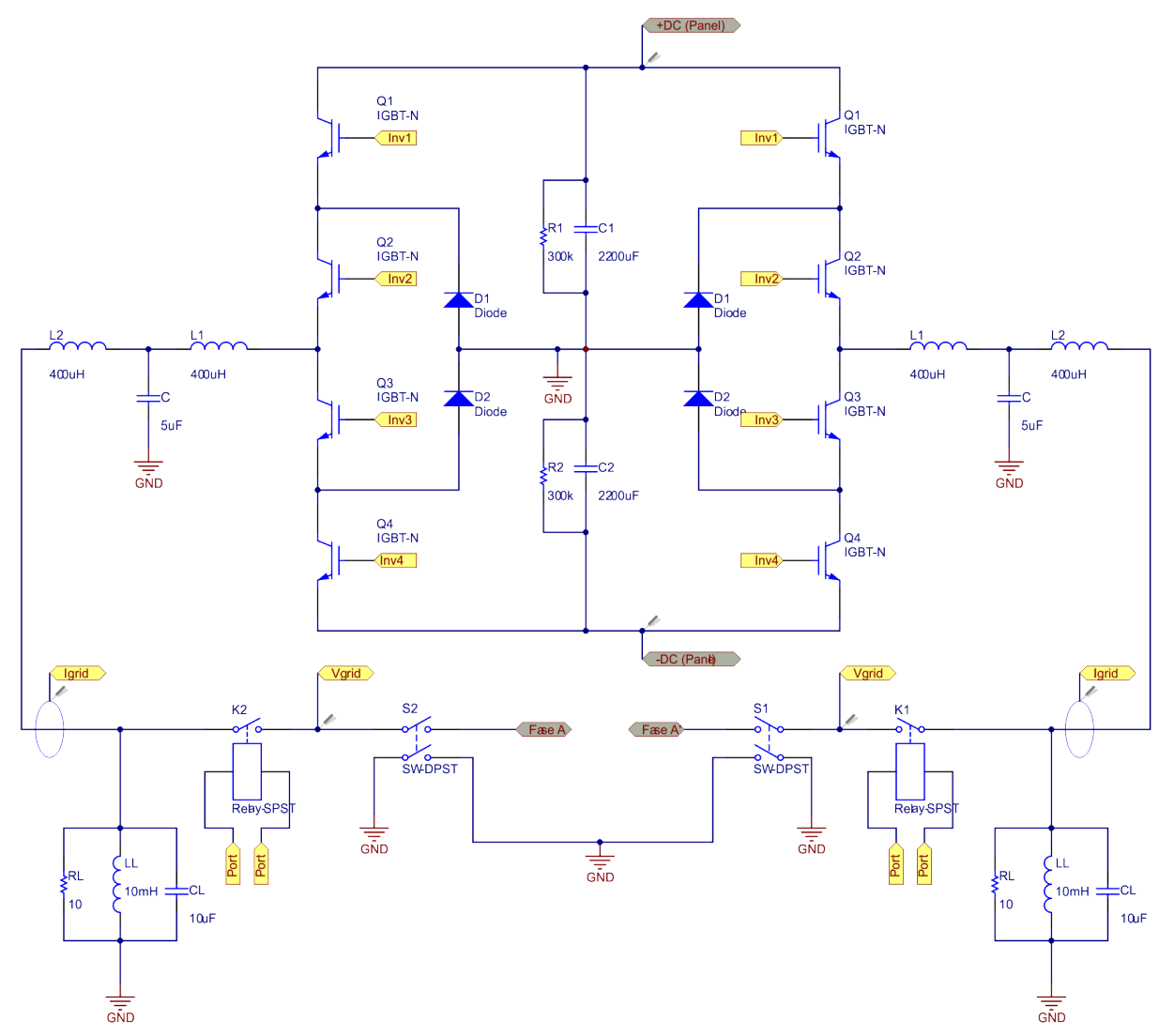

Figura 6. Circuito esquemático de las 2 ramas del inversor. 


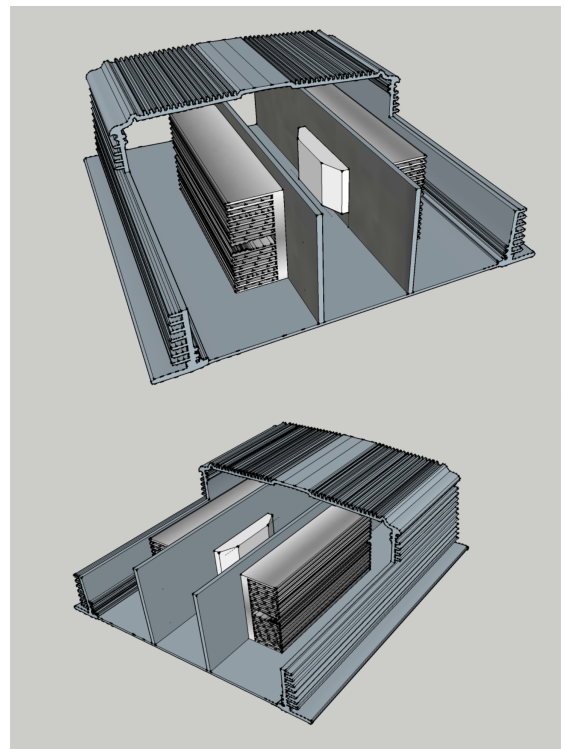

Figura 7. Disipadores y módulos IGBT dentro del gabinete de aluminio elegido.

\section{Simulación de la operación del sistema}

El funcionamiento de todo el sistema se prueba mediante un modelo desarrollado en MATLAB/Simulink. Esto permite realizar numerosos ajustes previos requeridos en el control de corriente, tipo de modulación, etc. Los cuales serían mucho más dificultosos de realizar directamente sobre el sistema real. El esquema de control desarrollado se basa en un algoritmo de MPPT que se encargará de extraer siempre la máxima potencia disponible en el panel fotovoltaico. Este algoritmo detecta el punto de operación en el cual se extrae la máxima potencia al panel y genera la ampli- tud de referencia de corriente que el inversor inyectará a las cargas domiciliarias y, de haber un excedente, a la línea eléctrica pública. La operación del inversor está pensada para ser a factor de potencia unitario, sin embargo, es factible operarlo a factores distintos de la unidad en caso de ser requerido. En este caso el inversor podría comportarse como filtro activo de potencia y el estudio en ese modo de funcionamiento está previsto en trabajos futuros. La Figura 8 muestra el esquema general de la simulación donde se pueden ver el bloque correspondiente al inversor NPC, el bus DC, y el filtro LCL de acoplamiento a la línea. Dentro del bloque de control se implementan todos los lazos requeridos para la operación del sistema así como un lazo de enclavamiento de fase (PLL, por sus siglas en inglés) que sirve como marco de referencia para la generación de fases y transformaciones vectoriales. El esquema mostrado incluye el control de solamente una fase ya que el control en operación bifásica resulta completamente simétrico.

La Figura 9 muestra la corriente entregada por el inversor para una referencia de 25 A lo cual equivale a una potencia de $3000 \mathrm{~W}$. Esta corriente surge del cálculo de disipación máxima que se realiza para cada IGBT dejando un margen de seguridad. Cada fase puede entregar esta corriente por lo tanto, la capacidad total del inversor resulta de aproximadamente $6000 \mathrm{~W}$ independientemente de que opere en configuración monofásica o bifásica. De la gráfica puede observarse que la corriente obtenida está completamente en fase con la tensión de línea y su distorsión armónica total (DAT) resulta de 2,9\%.

En el ejemplo anterior se supuso tensión de línea completamente sinusoidal lo cual no es el caso típicamente encontrado en redes reales. En la Figura 10 se muestra el resultado de la simulación cuando se modela una tensión de línea con una DAT de 5,5\%.
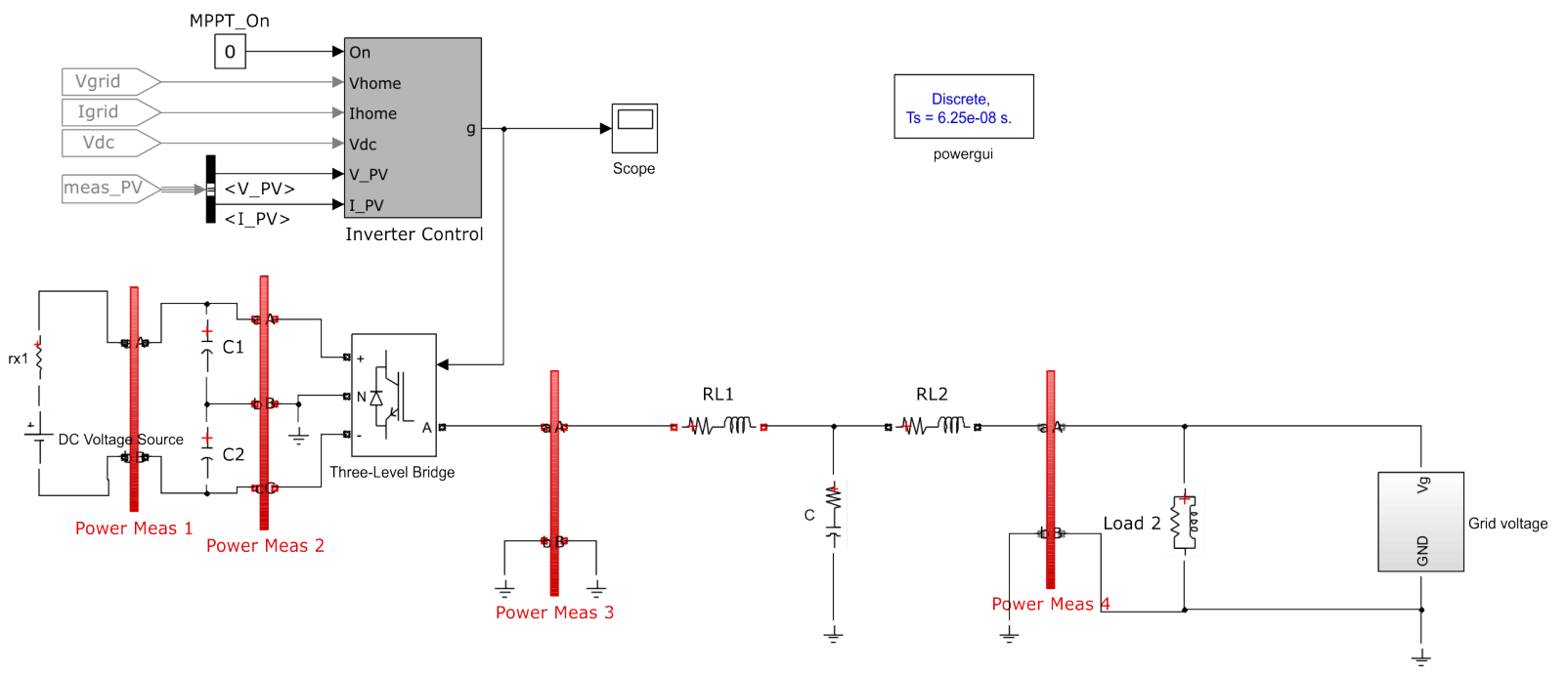

Figura 8. Modelo del inversor implementado en MATLAB/Simulink. 
En este caso se obtiene una corriente de línea aún en fase y con una DAT de 4,9\% mostrando que el control puede operar en condiciones no ideales de tensión dando incluso corrientes con DAT más bajas que la de la tensión de línea.

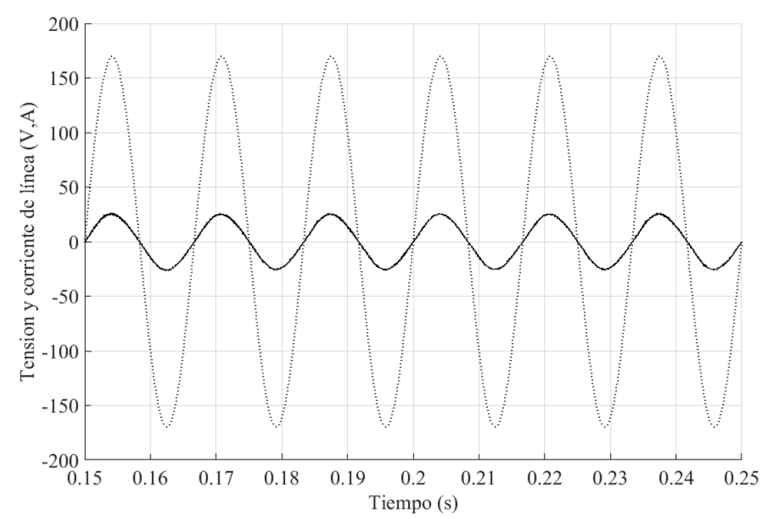

Figura 9. Corriente inyectada por el inversor y tensión de línea ideal.

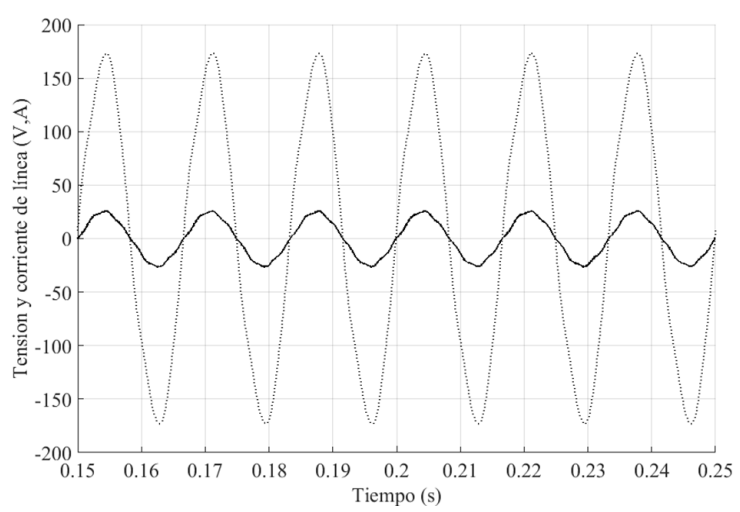

Figura 10. Corriente inyectada por el inversor y tensión de línea con DAT $=5,5 \%$.

\section{Resultados experimentales}

Se presentan algunos resultados preliminares obtenidos con un prototipo que se encuentra funcionando en los laboratorios del Grupo de Investigación en Energías de la Universidad Politécnica Salesiana. Para facilitar el análisis y medición de las variables involucradas el prototipo no se monta todavía dentro del gabinete tal como se muestra en la Figura 11. El sistema es controlado por una tarjeta basada en el microprocesador ADSP-21369 de Analog Devices el cual procesa en tiempo real la información suministrada por los sensores de corriente y tensión y genera la acción de control necesaria [12]. La frecuencia de conmutación se estableció en $16 \mathrm{kHz}$, al igual que la de adquisición. El filtro LCL tiene los mismos valores calculados en la sección 3.2 y utilizados en la simulación, esto es: $\mathrm{L} 1=\mathrm{L} 2=400 \mu H$ y $\mathrm{C}=5 \mu \mathrm{F}$.
En la Figura 12 puede verse la evolución de la tensión entregada por el inversor en el punto de acoplamiento con la carga estando ésta recibiendo potencia enteramente del inversor. En la Figura 13 se muestra la corriente inyectada por el inversor cuando se acopla a una tensión de línea con una DAT del $10 \%$. La amplitud de la corriente inyectada es de $11 \mathrm{~A}$ y la DAT resulta de $4,5 \%$.

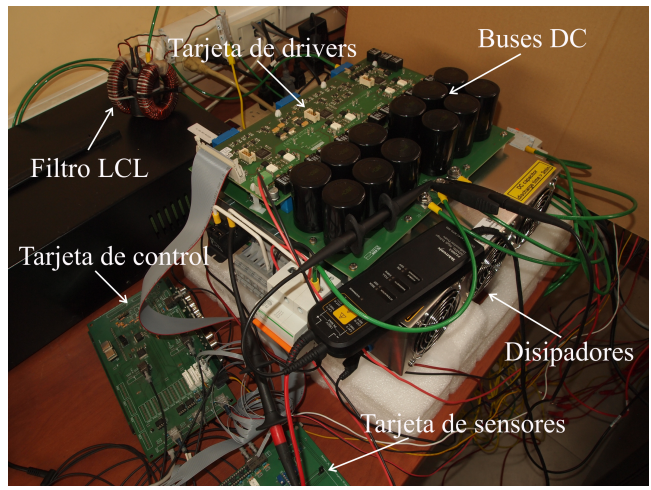

Figura 11. Imagen del prototipo utilizado para las pruebas experimentales.

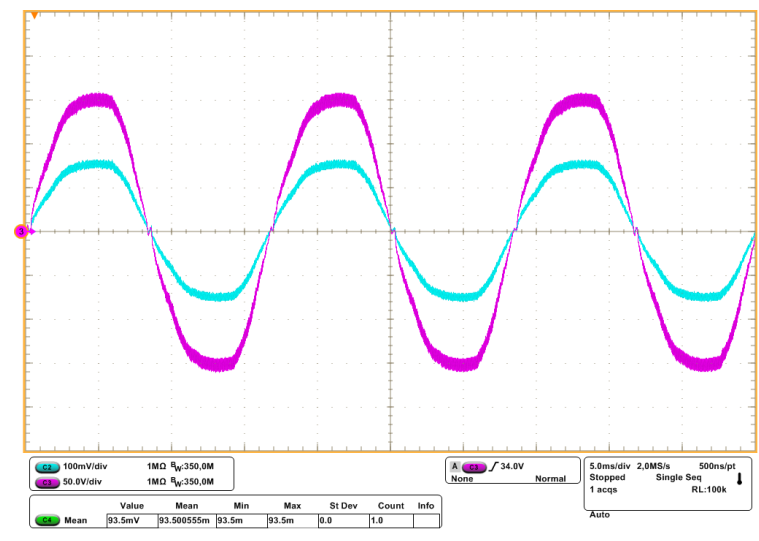

Figura 12. Tensión de salida del inversor (morado) y corriente en la carga (azul).

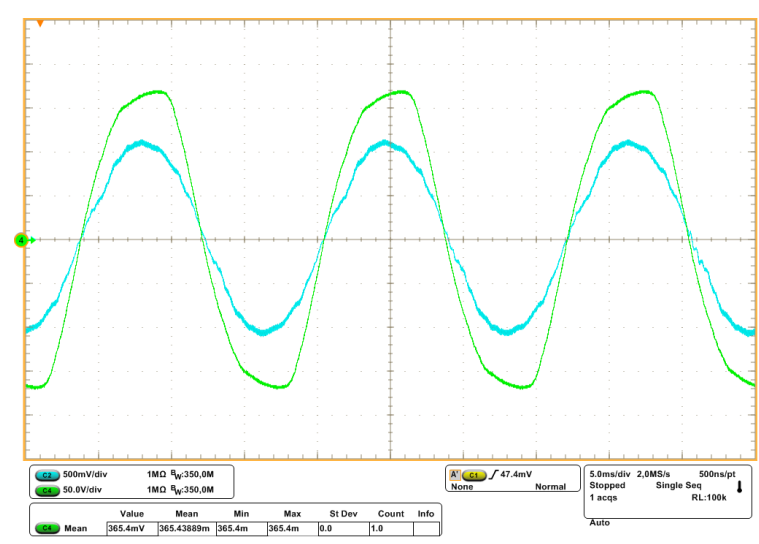

Figura 13. Corriente resultante (azul) cuando se acopla el inversor a una tensión de línea con DAT $=10 \%$ (verde). 


\section{Conclusiones}

El trabajo presenta el diseño y desarrollo de un inversor para aprovechamiento de la energía solar en instalaciones residenciales. Dentro de los criterios tomados en cuenta en el diseño se tienen la posibilidad de operar tanto en conexión monofásica como bifásica los cuales son los modos más comúnmente utilizados en la distribución eléctrica residencial en el Ecuador. En ambos casos puede suministrar una potencia de salida de $6000 \mathrm{~W}$.

La operación fue probada mediante simulaciones ante condiciones ideales y no ideales en la tensión de línea obteniéndose corrientes de línea con DAT menores incluso a la DAT de la tensión de línea. Los resultados experimentales preliminares muestran que el sistema puede operar de manera estable cuando alimenta una carga y al mismo tiempo inyecta potencia hacia la línea. La eficiencia del sistema ha sido estimada en $94 \%$ según las pruebas realizadas hasta el momento.

La operación típica del sistema diseñado es a factor de potencia unitario, sin embargo, futuros estudios prevén variantes en su control para poder operarlo con inyección de reactivos a la línea y actuar adicionalmente como filtro activo de potencia. El desarrollo presentado en este trabajo no incluye el uso de bancos de batería, pero se tiene contemplado la generalización del sistema para poder incorporar esa opción.

\section{Agradecimientos}

Los autores desean agradecer al Proyecto Prometeo, dependiente de la Secretaría de Educación Superior, Ciencia, Tecnología e Innovación (Senescyt), así como también a la Universidad Politécnica Salesiana por el apoyo institucional y financiero dado al desarrollo de esta investigación.

\section{Referencias}

[1] P. EnergyTrend. (2015, Sept) Price quotes updated weekly - PV spot prices.

[2] International Energy Agency. (2015) 2014 snapshot of global PV markets. International Energy Agency.
[3] IEEE Standard for Interconnecting Distributed Resources with Electric Power Systems, IEEE Application Guide for IEEE Std 1547(TM) Std. 1547.2-2008, 2009.

[4] IEEE Standard Conformance Test Procedures for Equipment Interconnecting Distributed Resources with Electric Power Systems, IEEE Std. 1547.12005, 2005.

[5] J. Viola and F. Quizhpi, "Desarrollo de un convertidor electrónico multinivel para aplicaciones de compensación de potencia reactiva," Revista Técnica Energía, no. 9, pp. 96-103, 2013.

[6] J. Restrepo, J. Viola, and F. Quizhpi, "Banco de emulación de perfiles de viento para aplicaciones en energía eólica," Revista Técnica Energía, no. 11, pp. 77-84, 2015.

[7] B. Babcsany, "Is the new german energy policy sustainable?" in 4th International Youth Conference on Energy (IYCE), 6-8 Jun 2013, pp. 1-4.

[8] R. Teodorescu, M. Liserre, and P. Rodriguez, Grid converters for photovoltaic and wind power systems. John Wiley \& Sons, 2011, vol. 29.

[9] A. Nabae, I. Takahashi, and H. Akagi, "A new neutral-point-clamped pwm inverter," Industry Applications, IEEE Transactions on, no. 5, pp. 518-523, 1981.

[10] Vincotech, Transistores de compuerta aislada, Vincotech. [Online]. Available: http: //www.vincotech.com/uploads/tx_st9products/ 10-FZ07NMA100SM-M265F58-D1-14.pdf

[11] J. Viola, J. Restrepo, F. Quizhpi, and J. Aller, "Predictive control of a three-phase power converter coupled with LCL filter," in IEEE International Conference on Industrial Technology (ICIT), 17-19 Mar 2015, pp. 2322-2326.

[12] J. Viola, J. Restrepo, F. Quizhpi, M. Giménez, J. Aller, V. Guzman, and A. Bueno, "A flexible hardware platform for applications in power electronics research and education," in Electrical Power Energy Conference (EPEC). IEEE, 1-6 Nov 2014. 